\title{
Transcriptional cytokine responses associated with pathological outcomes in chickens experimentally infected with pigeon variant of avian avulavirus type 1
}

\author{
Monika Olszewska-Tomczyk ${ }^{1 \S}$, Agnieszka Jasik² \\ ${ }^{1}$ Department of Clinical Sciences and Diagnostic, Institute of Veterinary Medicine, \\ Faculty of Biological and Veterinary Sciences, Nicolaus Copernicus University, \\ Gagarina 7, 87-100 Toruń, Poland. \\ olszewska.tomczyk@umk.pl, https://orcid.org/0000-0001-9188-3950 \\ ${ }^{2}$ Department of Pathology, National Veterinary Research Institute, \\ Al. Partyzantów 57, 24-100, Puławy, Poland. \\ agnieszka@piwet.pulawy.pl, https://orcid.org/0000-0003-3151-7267
}

Corresponding author: Monika Olszewska-Tomczyk, ${ }^{1}$ Department of Clinical Sciences and Diagnostic, Institute of Veterinary Medicine, Faculty of Biological and Veterinary Sciences, Nicolaus Copernicus University, Gagarina 7, 87-100 Toruń, Poland. E-mail: olszewska.tomczyk@umk.pl

\section{Abstract}

Background: Newcastle disease (ND) caused by virulent avian avulavirus 1 (AAvV-1) within the family paramyxoviridae, affects variety of avian species around the globe as well as responsible for significant economic losses in the poultry industry. 
Methods: Transcriptional response of selected cytokines associated with pathological outcomes were studied on chickens after experimental infection with three virulent isolates of pigeon variant avian avulavirus type $1(\mathrm{PAvV}-1)$. The specific pathogen free (SPF) chickens were used in the study, after virus inoculation via the intranasal route virus, observed daily for clinical symptoms. On day 3 of post infection, the experiment was terminated and then anatomopathogical and histopathological changes, virus load and transcriptional cytokine response were determined in the spleen.

Results: For the study, four cytokines were chosen: interferons (IFN- $\beta$, IFN- $\gamma$ ) and interleukins (IL-2, IL-6). The increased transcriptional levels of cytokine expression were associated with viral gene expression and marked lesions. The increase in the mRNA levels of IL- 6 was most significant in chickens with clinical symptoms. The combined up-regulations of IFN- $\gamma$ and IL- 6 mRNA expression were detected simultaneously with infiltration of macrophages in the histopathology analysis. The RNA expression of IL-2 and slight upregulation of IFN- $\beta$ were only in chickens infected with isolate closely related to poultry isolates in the phylogenetic study.

Conclusions: The difference in the mRNA expression levels of cytokines may help to elucidate mechanisms of immune responses in birds infected with different virulent strains of avian avulavirus type 1 .

Keywords: chicken; pigeon variant of avian avulavirus type 1; innate immune response; cytokine; pathogenicity; viral loads.

\section{Introduction}

Avian avulavirus type $1(\mathrm{AAvV}-1)$ cause contagious disease, that affect worldwide birds in all age. According to the world organization for animal health, it's a reportable disease in poultry when fulfilled criteria of virulence, resulting from determination of the intracerebral pathogenicity index (ICPI) or the amino acid sequence at the F protein cleavage site [1]. Infection of a variety of strains AAvV-1 initiated disease, that can have manifested differences in the severity of the symptoms. Based on virulence and clinical signs in chickens, there were distinguished pathotypes: viscetropic and neurotropic velogenic, mesogenic, lentogenic and asymptomatic forms [2]. In result of having a unique monoclonal antibody pro- 
file avian avulavirus type 1 isolated from pigeons are antigenically different from those detected in domestic poultry. In general, they are clustered in lineage $\mathrm{VIb} / 1$ of genotype $\mathrm{VI} \mathrm{AAvV-1}$ strains, in phylogenetic study [3]. Additionally, recent studies indicated, that the some of them are classified to group $4 \mathrm{a}$, which indicates relatedness to the $\mathrm{AAvV}-1$ isolated from poultry [4]. The AVVa-1's genetically related to pigeon variant are usually detected in their natural hosts. However, many cases of infection or isolation of the pathogen from other bird species including poultry, have been noted [5-7]. The ICPI value of isolates pigeon variant AVAs-1 are mostly greater than 1.0 and pathogenicity frequently characterized as from mild to intermediate virulence for domestic chickens [8]. Nevertheless, the occurrence of PAAvV-1 in poultry with ICPI equal to or greater than 0.7 or having a multiple basic amino acid motif at the $\mathrm{F}$ protein cleavage site is classified as virulent and should be reported to the OIE (CEC). The most serious outbreaks of virulent pigeon variant of $\mathrm{AAvV}-1$ in commercial chickens were documented in the UK during 1984, over 23 cases, whereas measures to control the spread of disease for these outbreaks resulted in the slaughter of over 800,000 chickens and expenditures of over $£ 2$ million in compensation and other costs [9-10]. However, the pathogenicity of PAAVv-1 for chickens was frequently absent, thus the role in poultry pathology still unclear [11-12].

Considering the fact of widespread of $\mathrm{PAAvV}-1$ in pigeons and yearly low number of reported occurrence in poultry in EU countries, interesting a molecular mechanism underlying of PAAvV-1 path infection in different species of birds. This study was conducted to extend knowledge about chicken response to infection of virulent strains of pigeon variant avian Avulavirus type 1. The transcriptional levels of mRNA of four cytokines, that play an important role in host antiviral response by stimulating the T-lymphocyte dependent system and being potent macrophage activation factors: IFN- $\beta$, IFN- $\gamma$, IL-2 and IL-6, were investigated. 


\section{Materials and Methods}

Viruses: For the experimental study three isolates were chosen, marked as: PPMV-1/PL/PW166-175/02, PPMV-1/PL/332/05, and PPMV-1/PL/ $\mathrm{H} 2 / 10$. All isolates were isolated from sick or dead feral pigeons from different parts of Poland over a 10-year period and had been characterized, details are listed in Table 1 [13-14].

Table 1. Background information of PPMV-1 isolates used in the study

\begin{tabular}{|c|c|c|c|c|}
\hline \multirow{2}{*}{\multicolumn{2}{|c|}{$\begin{array}{l}\text { Investigated } \\
\text { parameters }\end{array}$}} & \multicolumn{3}{|c|}{ Virus } \\
\hline & & $\begin{array}{l}\text { PPMV-1/PL/ } \\
\text { PW166-175/02 }\end{array}$ & $\begin{array}{c}\text { PPMV-1/PL/ } \\
332 / 05\end{array}$ & $\begin{array}{c}\text { PPMV-1/PL/ } \\
\text { H2/10 }\end{array}$ \\
\hline \multicolumn{2}{|c|}{ Mab 161/617 reactivity } & $1: 64$ & $1: 2048$ & $1: 32$ \\
\hline \multicolumn{2}{|c|}{ ICPI } & 0,61 & 1,05 & 1,29 \\
\hline \multicolumn{2}{|c|}{ Genotype } & $4 \mathrm{~b}$ & $4 \mathrm{~b}$ & $4 a$ \\
\hline \multicolumn{2}{|c|}{$\begin{array}{c}\text { F1/F2 cleavage site, } \\
\text { aa motif }\end{array}$} & ${ }^{112} \mathrm{R}-\mathrm{R}-\mathrm{Q}-\mathrm{K}-\mathrm{R}^{\star} \mathrm{F}^{117}$ & ${ }^{112} \mathrm{R}-\mathrm{R}-\mathrm{Q}-\mathrm{R}-\mathrm{K}^{\star} \mathrm{F}^{117}$ & ${ }^{112} \mathrm{~K}-\mathrm{R}-\mathrm{Q}-\mathrm{K}-\mathrm{R}^{\star} \mathrm{F}^{117}$ \\
\hline \multirow{2}{*}{$\begin{array}{l}\mathrm{L} \text { protein aa } \\
\text { at position }\end{array}$} & $-1564:$ & $\mathrm{N}$ & $\mathrm{N}$ & $\mathrm{T}$ \\
\hline & $-1694:$ & V & V & V \\
\hline
\end{tabular}

Briefly, two of the isolates, PPMV-1/PL/332/05 and PPMV-1/PL/ PW166-175/02, were identified as virulent, based on an ICPI value of greater than 0.7 and an amino acid sequence at the Fusion protein $(\mathrm{F})$ cleavage site. PPMV-1/PL/PW166-175/02 had a slightly lower ICPI index (ICPI= 0.61), but the amino acid sequence at the cleavage site of F1/ F2 typical of virulent strains. The phylogenetic analysis of nucleotide sequences of $\mathrm{F}$ gene each isolates indicated, that PPMV-1/PL/332/05 and PPMV-1/PL/PW166-175/02 belong to the $4 \mathrm{~b}$ sublineage as typical pigeon variants and closely related to other PAAVv-1 isolated in Poland. Additionally, PPMV-1/PL/H2/10 is atypical in phylogenetic analyses, belong to the genetic sublineage $4 \mathrm{a}$ closely related to $\mathrm{PAAvV}-1$ isolates from poultry. 
The sequence analysis of $\mathrm{L}$ proteins of the viruses revealed a lack of molecular markers associated with an increased virulence in chickens, but a unique amino acid profile was found in PPMV-1/PL/H2/10 [13]. Prior to the experiment, stocks of the viruses were prepared in 10-day-old SPF embryonated eggs (VALO BioMedia, Germany) according to standard procedure. Virus titers were calculated from the results of inoculation of 10-day-old embryonated eggs with a serial 10 -fold dilutions of the virus in phosphate-buffered saline (PBS).

Experimental design: SPF layer chickens were hatched from embryonated eggs purchased from VALO-BioMedia (VALO BioMedia, Germany). Twenty 3-week-old chickens were used in the study. Birds were divided into 4 groups. Three groups of five chickens were inoculated bilaterally both intranasal and via the conjunctival route with a $10^{6}$ median embryo infectious dose $\left(\mathrm{EID}_{50}\right)$ at $0,1 \mathrm{ml} /$ bird with different isolates of PPMV-1. The mock group received PBS. The birds were observed daily for clinical signs. The experiment was terminated at day 3 post infection (dpi), all birds were humanely sacrificed and their spleens were removed aseptically to determine histopathological changes, viral load and cytokine gene expression. The animal experiments performed in this study were approved by the II Local Ethics Committee of Animal Experimentation in Lublin (Permit number: 95/2015) in accordance with European and national regulations on animal experiments and welfare.

Histopathological examination: Spleens from infected and mock groups were immediately fixed after postmortem examination in $10 \%$ buffered formalin, embedded in paraffin, sectioned at $4 \mu \mathrm{m}$ and stained with haematoxylin and eosin (H\&E).

Immunohistochemistry (IHC): To detect PPMV-1 antigen in spleens, immunohistochemistry was performed on samples from infected birds. After deparaffinization, tissue sections were subjected to heat induced antigen retrieval by incubation in citrate buffer at $\mathrm{pH} 7.6$ for $20 \mathrm{~min}$ in a preheated pressure cooker to expose antigenic sites. Endogenous peroxidase 
blocking was done with $3 \%$ hydrogen peroxide diluted in methanol. The primary antibody (rabbit monoclonal, provided by Steven Essen, APHA, GB) was used at 1:500 dilutions for $60 \mathrm{~min}$ at room temperature. Staining was performed using labelled streptavidin-biotin visualization system kit, Dako REAL ${ }^{\mathrm{ru}}$ Detection Systems (Dako, USA). For visualization, 3,3'-diaminobenzidine-4HCl (Dako, USA) was used. Sections were counterstained with Mayer's haematoxylin, dehydrated and mounted. Sections incubated without the primary antibody and the use of negative tissues from non-infected chickens confirmed specificity of staining.

Quantification of viral RNA: Collected spleens from all birds (100 mg) were homogenized separately in $1 \mathrm{ml}$ of phosphate buffered saline (PBS) and centrifuged ( $2000 \times \mathrm{g} 15 \mathrm{~min}$ ). The RNA was extracted from $200 \mu \mathrm{l}$ of supernatant using commercial kit according to the manufacturer's instructions (RNasin, Qiagen, Germany). A quantitative one-step real-time RT-PCR (qRT-PCR) was performed with primers and probe specific to the matrix gene, designed by Wise et al. [15] using a QuantiTect Probe RT-PCR kit (Qiagen, Germany) in a $25-\mu \mathrm{l}$ reaction volume on an ABI 7500 Fast Real-Time PCR System (Applied Biosystems, USA). The cycle conditions of the RT and PCR steps were as follows: $50^{\circ} \mathrm{C}$ for $30 \mathrm{~min}, 95^{\circ} \mathrm{C}$ for $15 \mathrm{~min}$ and $40 \mathrm{cycles}$ at $95^{\circ} \mathrm{C}$ for $10 \mathrm{sec}, 56^{\circ} \mathrm{C}$ for $30 \mathrm{sec}$ and $72^{\circ} \mathrm{C}$ for $10 \mathrm{sec}$. The results of the qRT-PCR were converted from $\mathrm{Ct}$ values and expressed as eqEID ${ }_{50}$ per gram of tissue. Samples with a threshold cycle $(\mathrm{Ct})<36.0$ were considered positive.

Quantification of cytokine gene expression: The mRNA expression levels of IFN- $\beta$, IFN- $\gamma$, IL- 6 and IL-2 in spleens from infected and control chickens were analyzed using a two-step qRT-PCR with $\beta$-actin as a reference gene. Total RNA was isolated from $50 \mathrm{mg}$ of each spleen, following disruption in liquid nitrogen, using Total RNA Mini (A\&A Biotechnology, Poland) according to the manufacturer's instructions. Resulting RNA concentration was measured by determination of A260/A280 ratio in NanoDrop Spectrophotometer (Thermo Fischer Scientific, Germany) and ranged between 1005-1880 $\mathrm{ng} / \mu \mathrm{l}$. To prevent genomic DNA 
contamination, $1 \mu \mathrm{g}$ of total RNA per sample was treated with $1 \mathrm{U}$ of DNase I (Fermentas, USA) and used for reverse transcription reaction using $40 \mathrm{U}$ of $\mathrm{M}-\mathrm{MuLV}$ reverse transcriptase (Invitrogen, USA) with $50 \mathrm{ng}$ random primers (Invitrogen, USA) in the following conditions: $25^{\circ} \mathrm{C}$ for $10 \mathrm{~min}, 42^{\circ} \mathrm{C}$ for $60 \mathrm{~min}$ and $70^{\circ} \mathrm{C}$ for $15 \mathrm{~min}$. Relative changes in mRNA expression were measured using QuantiTect SYBR Green PCR Kit (Qiagen, Germany) and primers. The primers used in study were selected from previously published sequence [16-17], listed in Table 2.

Table 2. Primer sequences used in real-time PCR

\begin{tabular}{|c|c|c|}
\hline Target RNA & Primer sequence $\left(5^{\prime}-3^{\prime}\right)$ & $\begin{array}{l}\text { Product } \\
\text { size }(b p)\end{array}$ \\
\hline$\beta$-actin & $\begin{array}{c}\text { F:AGAGGCTCCCCTGAACCCCAAAGC } \\
\text { R:CTGGATGGCTACATACATACATGGCTGG }\end{array}$ & 94 \\
\hline IFN- $\beta$ & $\begin{array}{l}\text { F:GCCCACACACTCCAAAACACTG } \\
\text { R: TTGATGCTGAGGTGAGCGTTG }\end{array}$ & 150 \\
\hline IFN- $\gamma$ & $\begin{array}{c}\text { Forward:GTGAAGAAGGTGAAAGATATCATGGA } \\
\text { Reverse:GCTTTGCGCTGGATTCTCA }\end{array}$ & 71 \\
\hline IL-6 & $\begin{array}{c}\text { Forward:GCTCGCCGGCTTCGA } \\
\text { Reverse:GGTAGGTCTGAAAGGCGAACAG }\end{array}$ & 71 \\
\hline IL-2 & $\begin{array}{c}\text { Forward:TTGGAAAATATCAAGAACAAGATTCATC } \\
\text { Reverse:TCCCAGGTAACACTGCAGAGTTT }\end{array}$ & 93 \\
\hline $\begin{array}{l}\text { Virus matrix } \\
\text { (M) gene }\end{array}$ & $\begin{array}{l}\text { Forward:AGTGATGTGCTCGGACCTTC } \\
\text { Reverse:CCTGAGGAGGCATTTGCTA }\end{array}$ & 121 \\
\hline
\end{tabular}

Reactions were done in triplicate in $25-\mu \mathrm{l}$ volume containing $7.5 \mathrm{pmol}$ of each primer and $60 \mathrm{ng}$ of total RNA. Amplification and detection of the specific products were performed using ABI 7500 Fast Real-Time PCR System (Applied Biosystems, USA) with the following thermal profile: one cycle at $95^{\circ} \mathrm{C}$ for $15 \mathrm{~min}, 45$ cycles of $94^{\circ} \mathrm{C}$ for $15 \mathrm{~s}, 58^{\circ} \mathrm{C}$ for $30 \mathrm{~s}$ and $72^{\circ} \mathrm{C}$ for 30 s. At the end of each run a dissociation curve step was added and melting curve analyzed. 
Statistical Analysis. The relative expression of the target genes in the infected and mock group was calculated with the $2^{-\Delta \Delta \mathrm{Ct}}$ method. The housekeeping gene encoding $\beta$-actin was used as the reference against which to normalize the expression levels of the target genes. Statistical significance was calculated with the Mann-Whitney-Wilcoxon test. A probability (p) value $<0.05$ was considered statistically significant. All statistical calculations were computed in STATISTICA version 6.0 (StatSoft, Inc., USA).

\section{Results}

Clinical disease and pathology: At 3 dpi depression and slightly swollen tissues around the eyes were observed in chickens infected with PPMV-1/PL/PW166-175/02. In the same group, we noticed enlarged and friable spleen with many multifocal white lesions in $3 / 5$ birds (Figure 1). No clinical signs or pathological changes were observed in either of the two remaining groups.

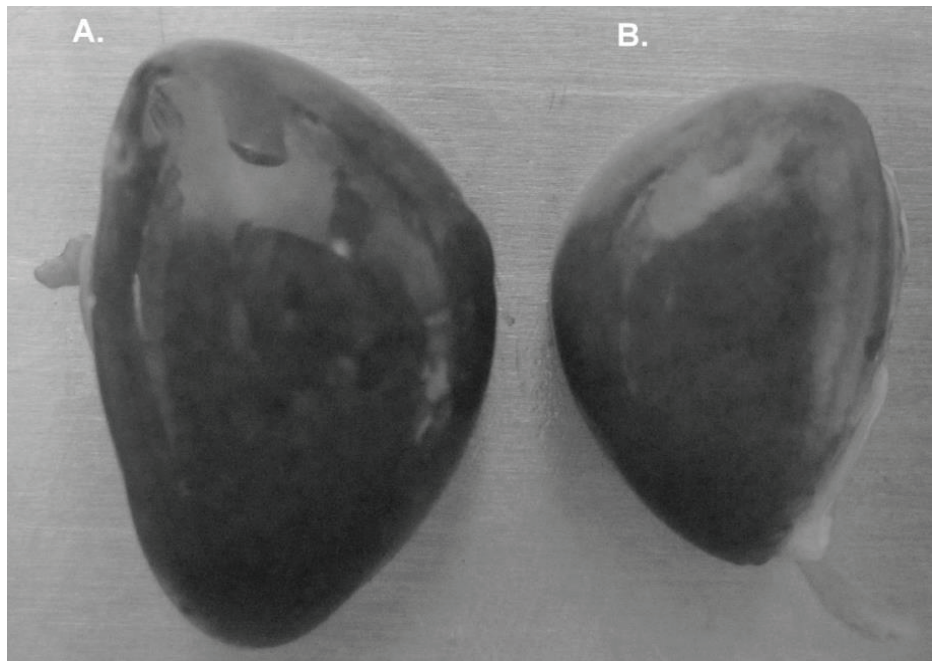

Figure 1. Images of 3 dpi spleens (A) spleen from chicken infected with PPMV-1/ PL/PW166-175/02, enlarged, mottled indicating multifocal necrosis, (B) spleen from chicken from mock group. 
Histopathology and Immuno-histochemical labelling: Microscopic lesions were observed in all infected birds, but these did not differ substantially irrespective of the isolate of PAAvV-1 used in the experiment. We observed only differences of the intensity of the histopathological changes (Table 3).

Table 3. Differences in intensity of histopathological lesions (HE) and immune-histochemical staining $(\mathrm{IHC})$ of spleen sections between the infected groups of chicken. HE grading: (++) -multifocal to coalescing areas of hyperplasia of EAC (ellipsoid-associated cells), (+) - multifocal hyperplasia of EAC, $(-)$ - no observed changes. IHC (immunohistochemistry): (+) - positive IHC labelling visible in over $50 \%$ of the tissue section, (+/-) - positive IHC labelling visible in less than $50 \%$ of the tissue section

\begin{tabular}{|l|c|c|c|}
\hline & $\begin{array}{c}\text { PPMV-1/PL/ } \\
\text { PW 166-175/02 }\end{array}$ & $\begin{array}{c}\text { PPMV-1/PL/ } \\
332 / 05\end{array}$ & $\begin{array}{c}\text { PPMV-1/PL/ } \\
\text { H2/10 }\end{array}$ \\
\hline HE & ++ & + & + \\
EAC hyperplasia & + & - & - \\
Mild lymphoid depletion & + & $+/-$ & + \\
\hline IHC & + & & \\
\hline
\end{tabular}

Histopathological examination revealed multifocal hyperplasia of ellipsoid-associated cells morphologically resembling macrophages in each of infected spleen. In the group PPMV-1/PL/PW166-175/02 the accumulation of macrophages and surrounding mononuclear cells was most prominent, the lesions were coalescing and accompanied by mild depletion of peri-arterial lymphoid sheaths (Figure 2).

The spleen tissue samples from a chicken infected with PPMV-1/ PL/PW166-175/02 showing coalescing accumulations of ellipsoid-associated macrophages and moderate depletion of lymphocytes $(B, D)$. The spleen tissue samples from a chicken in the mock group (A, C). Bar scale $=50 \mu \mathrm{m}$ 

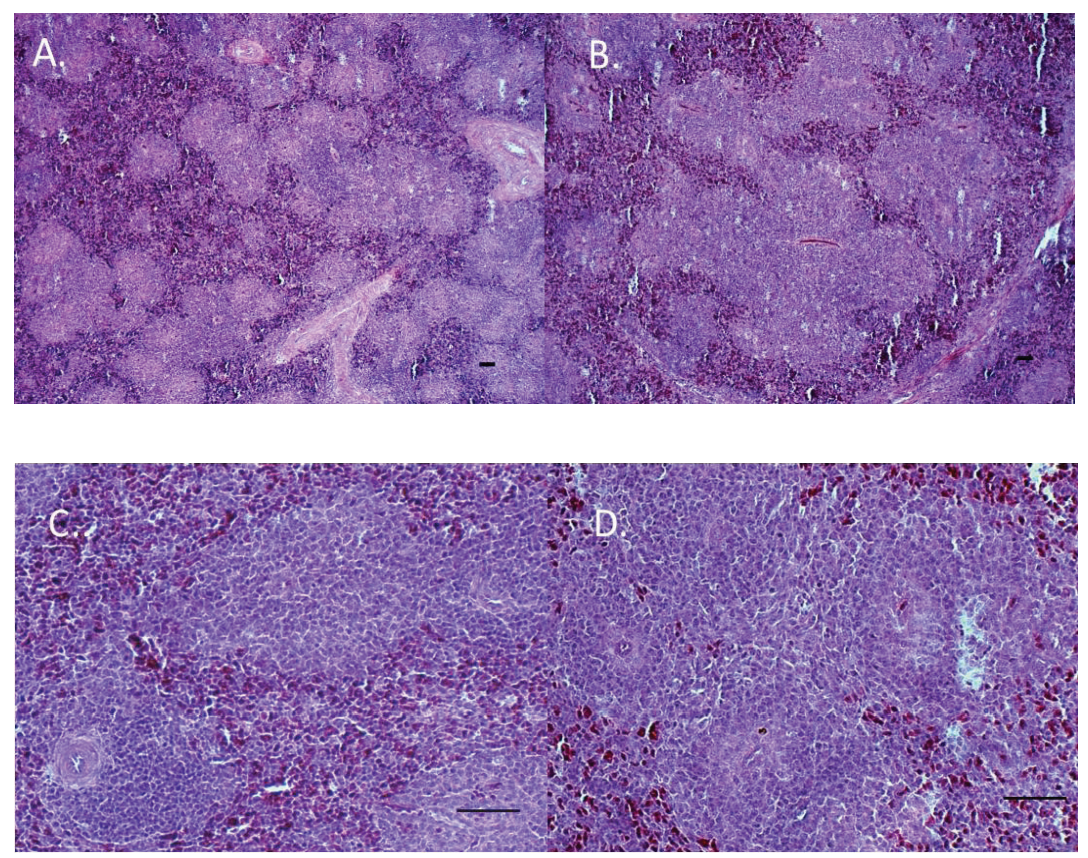

Figure 2. The histopathological changes in spleen

Furthermore, in all the groups, hyperaemia and occasional presence of fibrinous exudate in the sinusoids of spleen, as well as necrosis of ellipsoid-associated cells were observed. Immuno-histochemical labelling revealed positive reaction for viral antigen in all the infected spleens. The reaction was visible in the cytoplasm of mononuclear cells and sheaths of large macrophage-type cells (Figure 3). The diffuse labelling was most intense in PPMV-1/PL/PW166-175/02 and PPMV-1/PL/H2/10 groups while tissues of PPMV-1/PL/332/05 group shown weaker, multifocal labelling.

The chicken infected with PPMV-1/PL/H2/10 isolate, 3dpi; the diffuse immune-histochemical reaction is visible in a cytoplasm of cells morphologically resembling macrophages and lymphoid cells. immunehistochemical avidin-biotin-peroxidase, hematoksylin counterstain. Bar scale $=50 \mu \mathrm{m}$ 


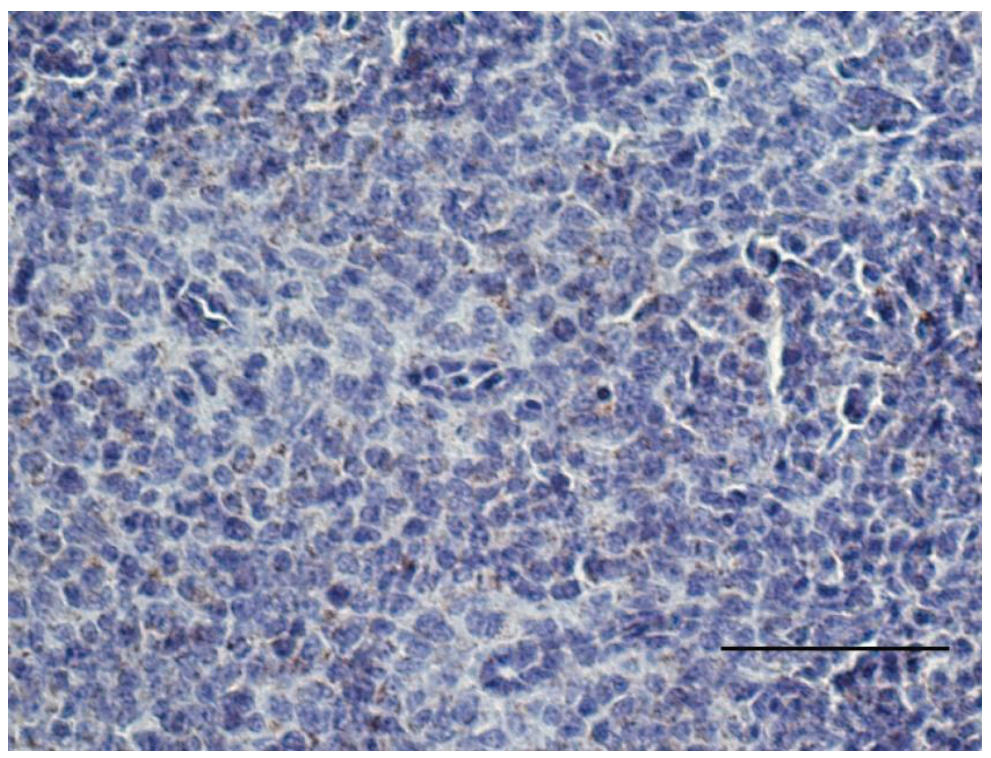

Figure 3. The immune-histochemical changes in spleen

Real-time RT-PCR: As illustrated in Figure 4, the viral RNA was detected in all spleens from infected chickens, but the number copies were not identical in spleens within the group. The amount of viral RNA reached its highest level in spleens from chickens infected with PPMV$1 / \mathrm{PL} / \mathrm{H} 2 / 10$, as the estimated median of equivalent titers in this group ranged from $2.96 \times 10^{5} \mathrm{eqEID}{ }_{50} / \mathrm{mg}$ to $2.07 \times 10^{6} \mathrm{eqEID} \mathrm{E}_{50} / \mathrm{mg}$. The lower viral RNA load, ranging from $4.9 \times 10^{4} \mathrm{eqEID}_{50} / \mathrm{mL}$ to $5.4 \times 10^{5} \mathrm{eqEID}_{50} / \mathrm{mg}$ was observed in spleens from the group of chickens infected with PPMV-1/ PL/PW166-175/02 and the lowest was found in spleens infected with PPMV-1/PL/332/05 and this ranged from 1.1 to $4.8 \times 10^{4} \mathrm{eqEID}{ }_{50} / \mathrm{mg}$. 


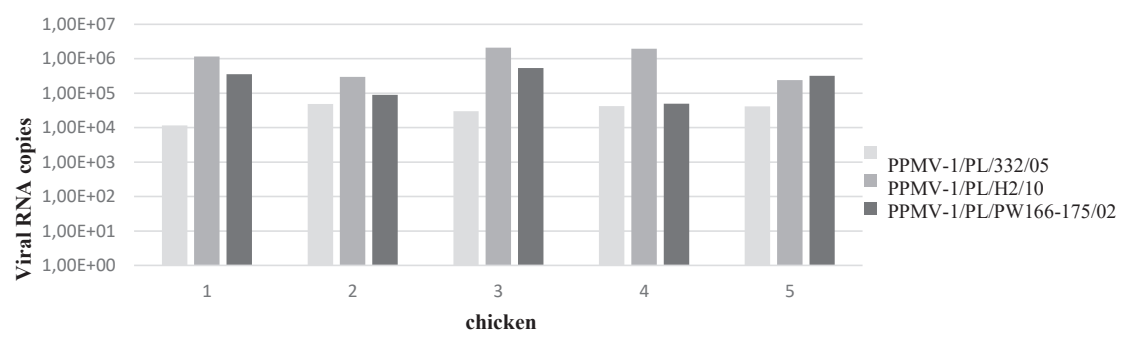

Figure 4. The eqEID 50 in spleen at $3 \mathrm{dpi}$ from chickens infected with different PPMV-1 isolates

IL-6, IL-2, IFN- $\boldsymbol{\gamma}$, IFN- $\beta$ mRNA relative expression: Relative changes in cytokine expression are presented graphically in Figure 5.
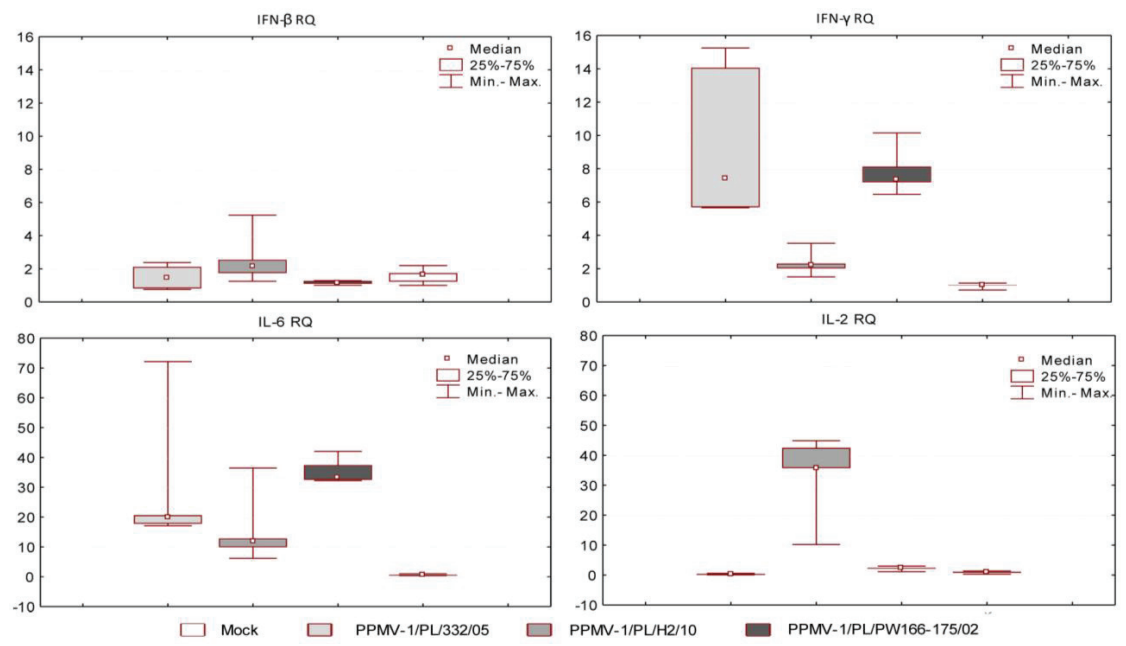

Figure 5. Determination of mRNA relative expression of IFN- ?? IFN-? IL-6, IL-2 genes in the spleen of chickens infected with different isolates of PPMV-1 calculated with the $2^{-\Delta \Delta} \mathrm{Ct}$ method

The changes in gene expression were compared to the mock group with the Student T test. The results showed that IL-6 mRNA relative expression (RQ) was upregulated and this increase appeared to be the most significant compared with other tested cytokines. The most marked upregulation 
of IL-6 expression was observed in chickens infected with PPMV-1/PL/ PW166-175/02 compared to the mock group (33.38 vs. 0.48) $(\mathrm{p}=0.00005)$. IL-6 transcriptional expression in birds infected with PPMV-1/PL/332/05 and PPMV-1/PL/H2/10 was also increased but to a lesser extent (20.07 vs. $0.48)$. This difference was statistically significant $(\mathrm{p}=0.000007)$. The analysis of IL-2 showed several higher RQ (35.83 vs. 1.00), but only in chickens infected with PPMV-1/PL/H2/10 ( $\mathrm{p}=0.0000005)$. Relative IL-2 RNA expression did not appear to change in chickens infected with the two other isolates. Upregulated levels of IFN- $\gamma$ gene expression were observed in each spleen from the infected groups. Similar levels were observed in both cases: PPMV-1/PL/332/05 and PPMV-1/PL/PW166-175/02 (7.42; 7.35 vs. 1.01) $\mathrm{p}=0.000003$. In comparison, the IFN- $\beta$ RNA relative expression levels were less remarkable and only in chickens infected with PPMV$1 / \mathrm{PL} / \mathrm{H} 2 / 10$ (2.15 vs. 1.6$)$ was there any statistical significance $(\mathrm{p}=0.03)$.

\section{Discussion}

Newcastle disease is one of the most important infectious diseases in poultry, and it causes significant economy impact to the poultry industry [18]. Virulent pigeon type 1 paramyxoviruses are dangerous for pigeons, but can also cause infections in other domestic birds. The pathogenicity of Newcastle disease viruses (NDV) is determined by the amino acid sequence of the precursor fusion protein protease cleavage site and cleavability at this site by the cellular protease [2]. However, NDV isolates that share the same F cleavage site have been recovered from many different species of birds and these isolates may produce mild or severe pathogenesis or even death [10]. Interestingly, some pigeon variants of NDVs, where the F protein is typical for virulent NDV strains, cause only minimal disease and show a low virulence in chickens [19]. Moreover, replacement of the $F$ gene of a virulent NDV strain with that of a non-virulent PPMV-1 strain does not affect the virulence of the host, thus indicating that the non-virulent PPMV-1 strain must be determined by other factors $[12,20]$. Consequently, it is highly desirable to conduct more research to 
explain the mechanism which contributes to the virulence of NDVs and its ability to cause differing pathogeneses in the host $[4,21]$. Although many studies have been performed to evaluate virus pathogenicity, evidence focusing on differences in virus-host interplay with different virulent isolates of NDV is limited [22].

Immediately following a viral infection, a host pro-inflammatory response is initiated. The first line of defense against a virus is innate immune response, which is the primary mechanism for resisting and clearing viruses during the early stage of infection [16]. Avian cytokines, like their mammalian equivalents, are influential in innate immune response to pathogenic infection [23]. Their appearance contributes to the reduction of viral replication, leads to the limitation of viral spread, and thus is associated with viral pathogenesis and pathological outcomes in the host. Interestingly, the induction of some cytokines may play a major role in the nature of the response to genetic variants of the pathogen, like in the case of serovars of Salmonella enterica in chickens [16]. Invasion of chicken cells by serovars $S$. Typhimurium or $S$. Enteritidis results in an increase of IL- 6 mRNA determined by quantitative RT-PCR. In contrast, invasion by the avian serovar S. Gallinarum does not lead to an increase of IL-6 mRNA [16]. The role of cytokines in the pathogenesis of NDV infections has not yet been extensively studied. There have been very few studies concentrating on immune response in chickens infected with NDV. Research has focused mostly on response to virulent NDV infection. In the study carried out by Ecco et al. [17], transcriptional IFN- $\beta$, IFN- $\gamma$, IL-2, IL-6 cytokine responses were studied in archived fixed, paraffin-embedded (FFPE) spleens from 4-week-old White Leghorn chickens from different pathogenesis experiments. They observed marked upregulation of INF- $\gamma$ and IL- 6 relative expression at 2 and 3 dpi in spleens from chickens infected with traditional velogenic and atypical velogenic viscetropic, and mesogenic of strains of Newcastle Disease. The birds inoculated with either of the two velogenic visectropic viruses at 3 dpi had evident clinical disease: histologically the spleen had evidence of necrosis in the lymphoid area. Interestingly, most significant upregulation of IL-6 was observed at 3 dpi in spleens of chickens infected with mesogenic strains of NDV, but 
clinical signs were not observed in those birds during experimental infection. Histologically, there was mild follicular hyperplasia and depletion in the spleen at 3 dpi. Relative IL- 2 and IFN- $\beta$ mRNA expression did not appear to change for either velogenic or mesogenic strains. Similar findings have been reported by other researchers [24] in relation to innate response in chickens infected with highly virulent NDV strains from the 2002 outbreak in California. Measurement of early host response to NDV in vivo in microarray experiments showed induced multiple gene signals at $48 \mathrm{~h}$ post infection in the Toll-like receptor which initiated IFN- $\alpha$ and IFN- $\beta$ expression. In an in vitro study, Rue et al. [24] characterized the early host response of splenocytes infected with virulent CA-NDV and lentogenic LaSota strains, showed that the lentogenic strains of NDV is not capable of rapidly and strongly inducing IFN- $\alpha$, IFN- $\gamma$ IL- 6 and IL- $1 \beta$, genes integral to pro-inflammatory response in contrast to virulent CANDV. The understanding of chicken innate response to Newcastle disease viruses is limited and, to our knowledge, there is no data regarding immunological response during infection with PPMV-1. In the present study, we investigated transcriptional expression of four cytokines - IFN- $\alpha$, IFN- $\gamma$, IL-2 and IL- 6 - in spleens of chickens at 3 dpi after infection with three different isolates of PPMV-1. The spleen was chosen for analysis of the early innate host response, because it is a lymphoid organ that the virus reaches early in Newcastle disease [22, 25]. It has been shown in multiple studies that macrophages and also lymphocytes play a crucial role during NDV infection [26]. Several major cell types present in the spleen (macrophages, heterophils, B-cells and T-cells) which means that this appears to be a promising organ for studies into immune response after NDV infection [27]. Macrophages have a distinct role in host response against viral infection. They promote clearance of virus-infected cells, and act as antigen presenting cells expressing pattern recognition receptors (PRRs) that recognize foreign molecules (pathogen associated molecule patternsPAMPs). The activation of PRR by PAMP triggers the activation of transcription factors and the expression of innate antiviral genes such as interferon, which is the most significant factor for host antiviral defenses. The Newcastle disease virus can induce alpha (INF- $\alpha$ ) and beta (IFN- $\beta$ ) 
interferons in macrophages and gamma (IFN- $\gamma$ ) interferon mRNA in peripheral blood monoclear cells. Our data are consistent with some previous findings. The histopathology studies provide a good visual correlation to PCR assays of IFN- $\gamma$ expression levels. Interferon- $\gamma$ produced by $\mathrm{T}$ helper cells is a potent activator of macrophages, enhancing destruction of cell-associated pathogens [28]. We showed statistically significant differences in relative upregulation of IFN- $\gamma$ genes in all spleens of infected chickens. The highest level of expression corresponded with the higher intensity of the histopathological changes, but was not connected with the highest viral load in the spleen tissue. We did not observe this correlation in the case of relative gene expression of IFN- $\beta$ in any chickens from infected groups. IFN- $\beta$ is produced mainly by macrophages, which helps to protect neighboring cells from viral infection. We demonstrated an increase only in chickens infected with atypical strains of PPMV-1, where we detected the highest viral load. For our study, we chose IL-6 comes from activated macrophages, fibroblasts, and endothelial cells and serves to enhance vascular permeability and stimulates recruitment of inflammatory cells to the affected site. In this study, IL-6 RNA levels were notably increased in all the viruses of PPMV-1, but most especially in the case of typical strains of PPMV-1 which cause clinical signs of pathological changes in the spleen of infected chickens. The results suggest that increases in IL-6 expression play an important role in promoting inflammation and tissue damage in chickens infected with PPMV-1. The most significant differences of expression of the tested cytokines was demonstrated in IL-2 in each group. IL-2 is produced by T cells and is critical for the proliferation of a number of immune cells, including Thelper lymphocytes, NK cells and B lymphocytes. However, IL-2 can lead to apoptosis as well as proliferation, making it unclear whether IL-2 has a predominantly positive or negative effect. However, we did not observe clinical signs or severe changes in chickens infected with the atypical strain of PPMV-1.

Conclusions: In order to further our studies and to demonstrate the role of PPMV-1 in chicken pathology, more research focused on the determination of changes in the percentage of $\mathrm{T}$ and $\mathrm{B}$ lymphocytes and 
macrophages isolated from the spleen of chickens infected with PPMV1 should be performed, in particular for atypical strains. To summarize, we demonstrated increase IL- 6 and IFN- $\gamma$ cytokines with accumulation of numerous cells morphologically resembling macrophages, but not with the quantity of the virus. The atypical strain of PPMV-1 showed a clearly different profile for the tested cytokine compared to typical strains of pigeon variant Newcastle disease virus. The difference in the mRNA expression levels of cytokines may help to elucidate the mechanisms of immune responses in birds infected with different virulent strains of APMV-1 that cause distinguishable clinical courses and pathologic changes.

Acknowledgements: We would like to thank Elżbieta Juszczuk, Anna Wijaszka for their excellent technical assistance as well as the staff of the BSL $3+$ animal facility for taking care of the birds.

Conflict of interests' statement: The authors declare that there is no conflict of interests regarding the publication of this article.

Financial disclosure statement: This work was funded by KNOW (Leading National Research Centre) Scientific Consortium "Healthy Animal Safe Food", decision of Ministry of Science and Higher Education No. 05-1/KNOW2/2015.

Animal rights ethical statement: The experiment was approved by Local Ethics Commission (University of Life Sciences in Lublin, Poland).

\section{References}

[1] World Organisation for Animal Health. Newcastle disease. Terrestrial animal health code, (Internet: http://www.oie.int/en/international-standard-setting/terrestrial-code/access-online/ volume 2, 2013.

[2] Alexander, DJ, Senne, DA. Newcastle disease, other avian paramyxoviruses and pneumovirus infections. In: Salif, Y.M.,Fadly, A.M.,Glisson, J.R.,McDo- 
ugald, L.R.,Nolan, L.K.Swayne, D.E.(Eds.) Diseases of Poultry. Iowa state University Press, Arnes, 2008; 75-116.

[3] Aldous, EW, Mynn, JK, Banks, J, Alexander DJ. A molecular epidemiological study of avian paramyxovirus type 1 (Newcastle disease virus) isolates by phylogenetic analysis of a partial nucleotide sequence of the fusion protein gene. Avian Pathol., 2003;32:239-256.

[4] Śmietanka K, Olszewska M, Domańska-Blicharz K, Bocian AL, Minta Z. Experimental infection of different species of birds with pigeon paramyxovirus type 1 virus--evaluation of clinical outcomes, viral shedding, and distribution in tissues. Avian Dis., 2014;58:523-30.

[5] Capua, I, Manvell, RJ, Antonucci, D. Scaramozzino P. Isolation of the pigeon PMV-1 variant of Newcastle-disease virus from imported pheasants (Phasianus colchicus). Zentralbl. Vet. Reihe B 1994;41:675-678.

[6] Ujvari, DE, Wehmann, EF, Kaleta, O, Werner, V, Savic, E, Nagy, G, Czifra, $\mathrm{B}$, Lomniczi. Phylogenetic analysis reveals extensive evolution of avian paramyxovirus type 1 strains of pigeons (Columba livia) and suggests multiple species transmission. Virus Res., 2003;96:63-73.

[7] Toro, H, Hoerr, FJ, Farmer, K, Dykstra, CC, Roberts, SR, Perdue M. Pigeon paramyxovirus: association with common avian pathogens in chickens and serologic survey in wild birds. Avian Dis., 2005;49:92-98.

[8] Kommers, GD, King, DJ, Seal, BS, Brown. CC. Virulence of pigeon-origin Newcastle disease virus isolates for domestic chickens. Avian Dis., 2001;45:906-921.

[9] Alexander, DJ, Parsons G. Avian paramyxovirus type 1 infections of racing pigeons: 2 pathogenicity experiments in pigeons and chickens. Vet. Rec., 1984;114:466-469.

[10] Seal BS, King DJ, Sellers HS. The avian response to Newcastle disease virus. Dev Comp Immunol., 2000;24:257-68.

[11] Biancifiori, F, Fioroni A. An occurrence of Newcastle disease in pigeons: virological and serological studies on the isolates. Comp. Immunol. Microbiol. Infect. Dis. 1983;6:247-252.

[12] Dortmans, JC, Koch, G, Rottier, PJ, Peeters BP. A comparative infection study of pigeon and avian paramyxovirus type 1 viruses in pigeons: evaluation of clinical signs, virus shedding and seroconversion. Avian Pathol. 2011;40:125-130.

[13] Pikula, A, Smietanka, K, Minta Z. Antigenic and genetic characteristics of PPMV-1 isolated in Poland. In: Proceedings of the $17^{\text {th }}$ World Veterinary 
Poultry Congress, Cancun, Mexico, J. M. Bradbury and N. E. Horrox, eds, 2011;764-769.

[14] Smietanka, K, Minta, Z, Domanska-Blicharz, K, Majer-Dziedzic, B, Pochodyla A. Characterization of pigeon paramyxovirus type 1 strains isolated in Poland in 1988-2005. B. Vet. I. Pulawy, 2006;50:283-286.

[15] Wise, MG, Suarez, DL. Seal, BS, Pedersen, JC, Senne, DA, King, DJ, Kapczynski, DR, Spackman E. Development of a real-time reverse-transcription PCR for detection of Newcastle disease virus RNA in clinical samples. J. Clin. Microbiol., 2004;42:329-338.

[16] Wigley P, Kaiser P. Avian cytokines in health and disease. Brazil J Poult Sci. 2003;5:1-14.

[17] Ecco R, Brown C, Susta L, Cagle C, Cornax I, Pantin-Jackwood M, Miller PJ, Afonso CL. In vivo transcriptional cytokine responses and association with clinical and pathological outcomes in chickens infected with different Newcastle disease virus isolates using formalin-fixed paraffin-embedded samples. Vet Immunol Immunopathol, 2011;141:221-9.

[18] Alexander, DJ. Newcastle disease in the European Union 2000 to 2009. Avian Pathol. 2011;40:547-558.

[19] Meulemans G, van den Berg TP, Decaesstecker M, Boschmans M. Evolution of pigeon Newcastle disease virus strains. Avian Pathol., 2002;31:515-519.

[20] Dortmans JCFM, Koch G, Rottier PJM, Peeters BPH. Virulence of pigeon paramyxovirus type 1 does not always correlate with the cleavability of its fusion protein. J Gen Virol 2009;90:2746-2750.

[21] Guo, H, Liu, X, Xu, Y, Han, Z, Shao, Y, Kong, X, Liu S. A comparative study of pigeons and chickens experimentally infected with PPMV-1 to determine antigenic relationships between PPMV-1 and NDV strains. Vet. Microbiol., 2014;168:88-97.

[22] Kapczynski DR, Afonso CL, Miller PJ. Immune responses of poultry to Newcastle disease virus. Dev Comp Immunol., 2013;41:447-53.

[23] Kaiser P, Staheli P. Avian cytokines and chemokines. In: Schat A.K., Kaspers B., Kaiser P. Avian Immunology. $2^{\text {nd }}$ Edition 2014; pp.189-204.

[24] Rue CA, Susta L, Cornax I, Brown CC, Kapczynski DR, Suarez DL, King DJ, Miller PJ, Alfonso CL. Virulent Newcastle disease virus elicits a strong innate immune response in chickens. J Gen Virol. 2011;92:931-9.

[25] Brown C, King DJ, Seal B. Detection of a macrophage-specific antigen and the production of interferon gamma in chickens infected with Newcastle disease virus. Avian Dis. 1999;43:696-703. 
[26] Kommers, GD, King, DJ, Seal, BS, Carmichael, KP, Brown CC. Pathogenesis of six pigeon-origin isolates of Newcastle disease virus for domestic chickens. Vet. Pathol. 2002;39:353-362.

[27] Dalgaard TS, Norup LR, Pedersen AR, Handberg KJ, Jørgensen PH, Juul-Madsen HR. Flow cytometric assessment of chicken T cell-mediated immune responses after Newcastle disease virus vaccination and challenge. Vaccine., 2010;28:4506-14.

[28] Kaiser P, Rothwell L, Galyov EE, Barrow PA, Burnside J, Wigley P. Differential cytokine expression in avian cells in response to invasion by Salmonella typhimurium, Salmonella enteritidis and Salmonellagallinarum. Microbiology 2000;146:3217-3226. 